stethoscope as much may be learned of the conditions in of the circulation in the lungs as has been learned, at the aortic area, of the systemic circulation.

In diseases of the mitral valve, whether obstructive or regurgitant, all authorities agree as to the importance of the accentuation of the pulmonic second sound, in deciding as to the degree of obstruction in the pulmonary vessels. One may probably, with safety, go even further than most authors go, in the case of an apical systolic murmur, which has existed for some time, and say, given such a murmur, not transmitted greatly to the left, without enlargement of the cardiac area, and without accentuation of the pulmonic second sound, that probably no serious change has taken place in the valve. In other words, it is a crucial test when one is in doubt as to the character of an apical systolic murmur, and, I believe, not appreciated at its full value.

This sound is equally available in case of a mitral obstructive lesion in forming a correct judgment as to the degree of obstruction, and the degree of compensation as well. In case of doubt as to whether a presystolic murmur at the apex were due to mitral obstruction or to aortic regurgitation (Flint's murmur), the absence of marked accentuation of the pulmonic second sound would weigh decidedly in favor of the integrity of the mitral valve, although I have not found this point mentioned in any of the treatises upon this subject.

In chronic disease of the chest of whatever nature, a marked accentuation of the second sound at the pulmonic orifice is of grave prognostic import, indicating a decided obstruction to the circulation in the vessels involved, although indicating as well an hypertrophy of the right ventricle to meet the increased demand. Whether the obstruction be from the destruction of capillaries in emphysema, the contraction of the interstitial tissue in fibroid phthisis, or the combination of destruction of tissue, infiltration of the new products and possibly other processes in ordinary tuberculosis, it indicates serious trouble.

A knowledge of the value of this sign may at times save one from serious error, as in the following case, seen in consultation some years since: A girl, six years of age, had presented for five days all the rational signs of acute lobar pneumonia, but the attending physician had been unable, after repeated examinations, to locate the disease. Finally, fearing that some other trouble was setting in, he asked me to see the case with him.

After hearing the history I confidently expected to find the signs of pneumonia in the left chest, and was surprised to find the fronts entirely normal, so far as I could determine. Because of the child's weakness, it was proposed to me that the examination of the back be omitted, but, having noted a decided accentuation of the pulmonic second sound, and called the attending physician's attention to it, I insisted upon the complete examination, confident that anything of sufficient gravity to cause a decided increase in the tension in the pulmonic artery, must be discoverable upon careful examination. The back was found normal, however, with the exception of an area two inches in diameter near the lower angle of the left scapula; here very moderate dulness existed, but the ausculatory signs were marked, namely, bronchial respiration, bronchophony and moist râles. It was evidently an acute pneumonia which approached the surface of the lung only at this point, and, to one not fairly expert in physical diagnosis, not easy to discover. I admit, of course, that no physician should overlook such signs, but they were overlooked by the attendant in this case, and by a previous consultant. If the two had appreciated the meaning of the decided accentuation of the sound in question, they would probably have been led to make a successtul search for the cause of it.

Other instances of somewhat similar nature might be quoted, but this one will suffice for illustration.

I believe that most of the present generation of physicians in active practice were taught that the murmurs at the pulmonic orifice were not only rare, but of comparatively little importance. The effect of this teaching has been to lead many of us to entirely neglect all sounds at this orifice in ordinary examinations, for I have often seen its area passed by without so much as the touch of the stethoscope. Inasmuch as a proper idea of the amount of obstruction in the pulmonic circuit in pneumonia, and hence of the amount of work called for from the right ventricle-a most important factor in prognosis and in deciding upon the advisability of administering cardiac stimulants-can not be obtained without an examination of the sound in question; nor a correct judgment be formed, in a case of chronic bronchitis, as to whether there is beginning obstruction, and consequent hypertrophy of the right heart, from emphysema which has not yet developed sufficiently to be patent to percussion, it would seem to be proven, when the evidence we have heretofore adduced is considered, that this sound should be investigated in every day practice exactly as other accessible chest sounds are, the ease with which such examination can be made being a powerful recommendation of the procedure.

\section{THE CARE OF CARDIAC DISEASE DURING THE QUIESCENT PERIOD.}

Read in the Section on Practice of Medicine, at the Forty-seventh Anuual Neeting of the American Medical Association, held BY LOUIS FAUGERES BISHOP, A.M., M.D. NEW YORK.

The term "care of disease" is a very pleasing one as representing one of the most honorable offices of the physician. The old Homeric definition of the physician as the one who cured internal diseases and took care of that which he could not cure, always seemed very happy especially when coupled with the definition of the surgeon as the one who cured extermal diseases. It is a very practical question, indeed, how we shall manage cases of organic cardiac disease so that the patient may enjoy a prolonged and comfortable existence.

A great change of public and professional opinion as to the grave prognosis, which was formerly given in valvular disease, has occurred during the present century. The old feeling was that a person with chronic heart disease was certain to die in a moderate length of time and was in constant danger of sudden death. The more careful physical examination of modern times has modified this view to a great extent, but credit must also be given to the better care of such cases. Sudden death is uncommon except in aortic insufficiency, and in cases complicated by true angina pectoris. It may be that sudden death was more common at a period in medical history when the use of digitalis was habitually abused. 
Time will not allow even the consideration of pathology in a most cursory way.

We are apt to think of chronic cardiac disease as confined too strictly to the valves. This contrary to analogy of the same class of lesions in other parts of the body. When a joint becomes the seat of chronic arthritis, the connective tissue of the remainder of the limb is frequently also involved, and the muscles are often the seat of pain.

The care of cardiac disease during the quiescent period is the care of the myocardium; for the endocardium we can do but little.

The causes of hypertrophy and dilatation are important to be understood. We refer to hypertrophy which goes beyond that degree which is desirable in a heart with damaged valves. . Excessive hypertrophy is best avoided by a regular hygienic life and by systematically watching for any cause which might increase the burden of the circulation, and removing it by treating the cause, or if that is not possible by the systematic use of arterial dilators.

Dilatation is that condition which the heart reaches when struggling under the burden of a circulation it can no longer maintain on account of the weakened myocardium. Instead of a firm contraction almost emptying the heart at each stroke, the systoles only partially expel the blood, and symptoms of impaired circulation are immediately evident. Dilatation is usually gradually established, occurring at intervals, for a time from a sufficient cause, and then perhaps disappearing. However, cases of acute dilatation, even in those previously unaffected by cardiac disease, are not at all uncommon. This gradual onset of dilatation is commonly brought about by overwork, anxiety, long continued excitement, intercurrent disease, or some other cause of increased cardiac strain. The study of the pulse as indicating various plans of management in the course of a cardiac case during the quiescent period is not as easy as one might suppose. If we are familiar with the case, and familiar with the personality of the particular pulse, it is easy enough to detect danger in some marked change. We have all of us seen cases of valvular disease in which a good degree of health and functional activity was carried on for a long period of time, and yet a person examining the pulse, who was not familiar with its habitual irregularity, intermittance, or permanent extreme feebleness, would give a prognosis of immediate calamity. The mere objective weakness of the heart's action apart from the signs of passive congestion should not lead to too bad a prognosis.

The question of exercise is important and interesting. Judicious exercise may bring about compensation, and prolong the period of its existence, and the deference of that time when the cardiac lesion will overbalance the power of the myocardium.

Taking our patient at the time when a valvular lesion has been the result of endocarditis or atheroma and the heart muscle has assumed successfully its additional burden the question arises in the mind of the physician, or perhaps is put by the patient, what forms of exercise shall be taken from a sense of duty, or may be indulged in for pleasure. To this question a categorical answer is not possible. We have to consider the occupation of the patient, his surroundings and his habits. Often enough his daily occupation and life will be found well suited to his condition. Such would be the case of a man whose business required a superintendence of out-door work or the work of a factory, that is, work devoid of sudden demands of strength, and devoid of mental excitement. The other two classes are those who do not get sufficient exercise, and those who are called upon for a dangerous amount of physical exertion. Curiously enough the former often suffer as much as the latter. The patient who leads a sedentary indolent life develops very soon the lung effects of passive congestion. The blood stasis, due to the poor heart performance, is augmented by the physical stagnation which follows insufficient exercise. Thus we see early developed in these patients chronic gastritis, constipation and the train of symptoms best described as biliousness. They take cold easily and there is quickly established a chronic catarrhal tendency in the respiratory passages. For these patients systematic out-door exercise in spite of their permanent cardiac lesion is of enormous advantage. The form of exercise must be such as will be of interest to the patient, otherwise persons of this disposition will never persist in carrying it out. The introduction of the game of golf would seem to afford an unusual opportunity for just such cases. It requires movement from place to place in the open air, pleasant surroundings, and is devoid of dangerous excitement and exertion. In certain cases bicycling on level roads in quiet districts is to be recommended, but such persons must be trusted not to enter into speeding contests. Driving, walking and calisthenics with light apparatus are also suitable. The patient should be instructed as to the principles involved, that is, that the exercise should be carried on slowly, systematically and never to the point of producing dyspnea, and never under any circumstances must competitions of strength or speed be entered into. The third class of patients who carry exercise too far are often difficult to manage because frequently enough their occupation is the only source of livelihood, and can not be readily abandoned or changed. Fortunately these patients frequently develop an unexpected degree of compensation, and provided the labor is regular they may continue for many years without the final reckoning. Still the average life of such individuals is very much shortened. If possible, these patients must be trained to do their work in a deliberate manner, avoiding sudden strains, and as much as possible they must be watched carefully for the signs of failing compensation. In some instances, when no other resource seems to be available, they must be tided over at times by the use of digitalis. Provided the work is systematic, even though severe, it is extraordinary how well some of these patients get along. We have all of us seen men with nearly all the murmurs in the whole circle of valvular lesions, who carry on occupations some of us could not endure for a single day; but all of us have likewise seen the day of reckoning when these men of powerful muscular development and splendid physique, except for their cardiac lesion, have come to a state where they can hardly lift a finger without gasping for breath. So in maintaining compensation, judicious exercise is one of the most important factors.

In connection with exercise massage should be mentioned. It has been happily defined as a process in which the operator takes the exercise and the patient receives the benefit. The use of hydrotherapy to improve the general nutrition and circulation is something worthy of attention.

It is a pity that in this country, at least, it is so 
difficult to obtain a proper carrying out of these measures. The advocates of hydrotherapy have so exaggerated its therapeutic value, great as it may be, that one often hesitates to subject his patients to their care. It is hoped that the time will come when properly trained persons will be available at a moderate cost to carry out hydrotherapeutic measures and not exceed the limits of their sphere. A drawback of hydrotherapy is that when carried out as it should be it will take a great deal of time. Much of the benefit is lost if a patient can not rest after bathing. The effect of a warm bath upon congested organs accompanied by gentle rubbing and followed by a period of rest in a recumbent position can not but be beneficial when carried out under proper precautions. The importance of the care of the skin aside from any special system in chronic cardiac disease is very great. The the theory that dirt obstructs the pores, however pleasant to believe, is probably not true. The value of bathing as far as health is concerned is more indirect in its effect. It improves the nutrition of the skin and stimulates its action and the tone of the general circulation.

As to clothing, the whole surface of the body except the hands and face should be always enveloped by flannel, and in severe weather this should be very much heavier than is thought necessary for bealthy people.

One of the first considerations when we come in contact with such a case, is the advisability of telling the patient of the existence of the condition. To my mind in the great majority of cases this is best. It requires a good deal of patience to supply the patient with a sufficient amount of knowledge to make the explanation comprehensive and free from terror. The first point is to disabuse the patient of the popular superstition that organic heart disease is a common source of sudden death. The tendency of the day to speak of death as "heart failure" exaggerates the difficulty at the present time. It may truly be said of a case of valvular disease of the heart that sudden death is not at all probable, and it might be added that it would be preferable to the long-drawn-out death by the gradual involvement of the organs from passive congestion, dyspnea and the supervention of dropsy. It is important that the patients should know that they have a damaged heart, because their coopperation is absolutely necessary in carrying out the proper regimen. The use of drugs in these cases directly stimulating the heart's action is seldom necessary. If the heart is unable to do its work by the physiologic increase of activity with the hypertrophy that naturally takes place, it is far better by absolute rest of the patient to give the heart a chance to catch up by its natural force than to whip it up to its work by drugs. Sometimes there will be cases when circumstances do not allow a cessation of the routine work of the patient. In these cases digitalis will often remove the symptoms of the heart's deficiency. When we have the coöperation of the patient a plan of existence must be laid out as free as possible from overwork, worry, over-exertion, and dissipation.

Physical labor, if not such as causes sudden strains upon the heart's action, need not be interdicted; but all causes leading to undue excitement, or such as tend to a neurasthenic condition, must be especially avoided. A person with organic cardiac disease can often perform very hard mental or even physical labor, provided it is unaccompanied by excitement or worry. These cases do better in the country than in the city. Routine existence is more naturally attained and the purer air and quieter surroundings conduce to the even plane of existence which we are seeking. Whatever plan of life is made, the great point is regularity. This is none the less true in the average case because now and again instances come under our notice of persons with badly damaged hearts, who lead lives of activity and excitement and accomplish a prodigious amount of labor, and still survive. These patients live longer than might be expected, but nearly all of them pay the penalty by a period of collapse and suffering. These cases of severe valvular disease, who do not submit to a proper regimen, nearly always succumb in the end, however long deferred, to the disease-itself, while the others may, as far as the heart is concerned, maintain an even plane of health, and die after a very long time only from some other disease.

In the drug treatment of these chronic cases the use of iron is of great value. The tendency to anemia which exists in all chronic diseases has in this instance a very direct tendency to exaggerate the symptoms. Sometimes dyspnea is entirely relieved when the impoverished blood is brought up more nearly to par. The alimentary system must receive very careful attention. The tendency of passive congestion to produce damage is very much increased by any functional derangement. Constipation is a very great evil in these cases, and regularity of this function should be absolutely insisted upon. The means must be chosen according to the merits of each case.

Against the use of digitalis and drugs of this class in the stage of compensation we would enter a strong protest. The routine practice is now happily rare of giving digitalis on account of the presence of a murmur without regard to other necessities. Strophanthus has proved in our hands a very valuable drug in tiding over periods of failure of compensation. For long use it has not been so satisfactory as digitalis.

The use of drugs during the quiescent period must to a large extent be the drugs which we are pleased to call "hygienic" drugs, namely tonics, laxatives, and occasionally alteratives. Strychnin is at times indicated in nearly all of these cases. It stimulates the nervous system in every part of the body, and in that way affects favorably the functions of nutrition, motion and secretion. Digitalis may be used at times when the circulation seems to overburden the heart and when for any reason the better remedy, rest, can not be applied. In chronic heart disease in individuals with specific taint, occasionally brilliant results have been obtained by specific treatment, the heart tone being apparently much improved under the course of medication.

The occasional use of mercurial purges in patients with chronic cardiac disease, who habitually overeat, is very important.

The use of alcohol in chronic heart disease is often baneful in its effects, and it is extremely difficult for the patient to break the habit. In the ordinary drunkard it is really the heart that cries out for alcohol when an attempt is made to stop its use, though the effects of the depressed heart are referred to the stomach, brain and general circulation.

The habitual use of alcohol is also to be discouraged on the ground of causing unnecessary exhaustion of the heart muscle and a tendency to tissue degeneration throughout the body It is to be remembered 
that fat is as often an indication of misdirected nutrition or degeneration as it is of hypernutrition. On the other hand in emergencies there is no drug so useful as alcohol in developing quickly and strongly, and at the necessary moment, the latent powers of the heart.

A discussion of the management of chronic heart cases would not be complete at this time without some reference to the influence of thyroid activity upon the heart's action. Without going into a full discussion of the probable fact that exophthalmic goitre is due to a hyposecretion of thyroid products, and that many conditions resembling obesity with its accompanying sluggishness of heart's action and tissue metabolism are to some extent dependent upon an under secretion upon the part of the thyroid, we can not but feel like administering the thyroid gland, at least in an experimental way, to those cases of chronic heart disease in which the heart as a whole and the heart fibers seem to be impaired in their action by fat. One of the most distressing complications of chronic cardiac disease are the attacks of pain, whether or not we dignify these attacks by the name "angina pectoris." The treatment of the attacks when they occur is not to be discussed here. The patients who seem to be liable to such attacks should often systematically take iolid of potassium.

While we have gone over at some length the considerations which are to be reviewed in forming a plan of management for each case, still much remains for the domain of individual judgment. In these patients much must be considered besides the actual objective symptoms at the time of the examination. Hereditary tendencies are a factor which must never be overlooked. If several members of the patient's family have succumbed to cardiac disease at about the same period of life, it is always a grave element in prognosis. That mysterious force, or rather storehouse, which we call latent energy, can not be fathomed and its capacity estimated with any degree of certainty. We have all of us seen patients who had apparently reached a state where compensation seemed impossible, gradually climb to a state of fair health and comfort. We have also seen patients who had gone on well for a long period of time, without apparent cause, suffer a loss of balance and perhaps go very quickly to a fatal termination.

In emphasizing the importance of hygiene in the management of cardiac disease during the quiescent period there is no stronger warning than that a recurrence of valvular inflammation is sure, by extension as well as by ovezwork, to involve the heart muscle in a secondary degeneration. Anti-rheumatic treatment should be instituted whenever any return of inflam. mation is feared, and the case should, for a short time at least, be managed with absolute rest, and the bland diet suitable to the inflammatory stage.

In discussing this subject we have not considered very much the particular lesion which might be found in the valves upon examination. We can not lay down specific rules for the management of mitral insufficiency, another set of rules for mitral stenosis, though the same management would be much more efficient when the former is the predominant lesion than when the latter exerts the greater influence. We can not treat a valvular lesion clirectly. Our prime object is to keep nutrition of the heart us good as possible, and by this we mean the avoidance of degeneration and the avoidance of excessive hypertrophy.
The nervous mechanism of the heart, as representing the conservator of heart energy, must be treated with consideration.

It would be an interesting question to hare definitely decided whether the actual volume of blood is greatly influenced by any practicable degree of dry liet. It has always seemed to us that the mechanism whereby the volume of fluid is maintained at a certain normal level was so perfect that any attempt to alter the volume of the blood by putting that mechanism on the strain must be inevitably fruitless. It is different, however, with the practical reduction of volume obtained by the use of nitro-glycerin, and such drugs, which diminish the resistance and by relaxing the smallest vessels bring about a relative reduction in the volume of blood.

In these ways and by many other little details, with the coopperation of an intelligent patient who understands thoroughly his condition, we can care for and maintain an even condition of apparent health for many years. Far more satisfactory is such a procedure than the up-hill work of the treatment when compensation has not been maintained, a contest which however much prolonged has but one final termination.

\section{LYCOPERSICUM CARDIOPATHIA.}

Read in the Sertion on Practice of Nedicine at the Forry-seventh Anual Meeting of the American Medical Association, nt At]anta. Ga., May 5-8, $189 t ;$

BY W. T. ENGLISH, A.M., M.D.

PROFESSOR OF PHYSICAL DIAGNOSIS IN MEDICALDFPARTMENT OF WESTERN UNIVERSITY OF PENNSYIVANIA AND CONSULTANT IN CHEST DISEASES IN THE SOUTH SIDF HOSPITAL, PITTSBURG, PA.

The lycopersicum esculentum, natural order of solanaceae, indigenous to South America, is a vining plant with irregular sessated leaves, bearing fruit of various shapes and colors. It is also known as the "love apple," but more commonly is called the "tomato." It was introduced into America in the latter part of the sixteenth century as an ornamental natural curiosity, and was found only in the conservatories of the wealthy. On account of its peculiar beauty it was subsequently taken to Europe. The fruit was considered poisonous to the human, but was observed to be eaten by lower animals. This fact excited Yankee curiosity and it was tested and adopted as an article of diet in some sections of America. It was not until the beginning of the civil war that its acceptance as an edible food became general. Europe has not up to the present given it general welcome as a food. Its principle utility is that of a relish and an agreeable addition to sauces. However, some people regard it as a wholesome and desirable acquisition to the list of foods. Because of its low nutritive value it does not merit a place with substantive aliment. Analyses have placed it among the vegetables consisting of over 85 per cent. water. According to Chambers and other writers on dietetics it is a diluent and not a food. The fluid element has been found to consist of an acid. called by some acidum lycopersicum. Others have claimed the acids to be acetic and malic in combination. Notwithstanding the almost universal indulgence in this fruit there is a certain proportion to whom it acts as a poison. In some the symptoms are gradually developed; in others they become immediately alarming. Some cases show no immediate evidences. but the results are cumulative. Once the abnormalities appear they are liable to continue to increase as long as the fruit is ingested. Even in 